\title{
RECONTEXTUALIZACIONES Y ENSAMBLAJES: ABP Y MATEMATICAS UNIVERSITARIAS
}

Recepción: 11/10/2015 | Revisión: 18/01/2016 | Aceptación: 13/03/2016

\author{
Paola VALERO Ole RAVN \\ Aalborg Universitet Aalborg Universitet \\ paola@plan.aau.dk orc@learning.aau.dk
}

Resumen: Un problema común en el aprendizaje de las matemáticas universitarias es la brecha entre el formalismo y la abstracción y el uso de los mismos en un contexto específico como la ingeniería. Las competencias adquiridas a través del aprendizaje basado en problemas ( $\mathrm{PBL}$, en español $\mathrm{ABP}$ ), en el modelo pedagógico de la Universidad de Aalborg en Dinamarca puede iluminar cómo tender un puente para cerrar esta brecha. A través del análisis de los reportes finales de semestre que realizan estudiantes de primer ciclo de ingeniería, donde herramientas matemáticas se recontextualizan y ensamblan en el abordaje de problemas interdisciplinarios complejos, describimos y analizamos las competencias logradas por los estudiantes cuando realizan ese tipo de proyectos. Queremos mostrar cómo la actividad de los estudiantes dentro de este ambiente ofrece posibilidades de aprendizaje que van más allá de la clásica distinción entre el aprendizaje formal y las aplicaciones de las matemáticas.

Palabras clave: aprendizaje basado en problemas; mediación tecnológica; modelación.

\section{RECONTEXTUALIZATIONS AND ASSEMBLAGES:}

\section{PBL AND MATHS AT UNIVERSITY}

Abstract: A common problem in university mathematics education is the gap between formalism and abstraction and how these two are applied in a specific context, for example, in engineering. The competencies gained through learning in a PBL environment, such as the one found at Aalborg University in Denmark, cast light on how to bridge such gap. We analyse, in depth, two first year engineering students' final project reports where mathematical tools are recontextualized and assembled in tackling complex interdisciplinary problems. The description and analysis of these cases allow us to illustrate the competencies that students gain when working on this type of project. We intend to show how students' activity in this environment offers possibilities that go beyond the classic divide between formal learning and application.

Keywords: problem based learning; technological mediation; modelling. 


\section{Introducción}

En la educación universitaria, las matemáticas son una asignatura especial que tradicionalmente ha sido presentada a los estudiantes como una entidad muy bien organizada con currículos y planes de estudio bien definidos. Los cursos de matemáticas a menudo se construyen sobre la estructura axiomatizada de las teorías matemáticas en cuestión. En un enfoque axiomatizado para el aprendizaje de las matemáticas se acostumbra a presentar una porción particular de las matemáticas partiendo de ciertos supuestos básicos para llegar a más y más verdades por medio de demostraciones. La presentación axiomatizada de los nuevos temas matemáticos a los estudiantes en forma de presentaciones magistrales es el modelo privilegiado para la enseñanza de las matemáticas. Algunas veces, las clases magistrales se complementan con sesiones de ejercicios o resolución de problemas donde el profesor o un asistente aclaran las dudas de los estudiantes al enfrentar los ejercicios del libro o los que propone el profesor. En este enfoque, el estudiante debe preocuparse por entender las estructuras de conceptos y procedimientos presentadas por los profesores; y algunas veces hay la oportunidad de apoyo en este proceso. Este tipo de patrón de enseñanza y aprendizaje domina en la mayoría de las aulas de matemáticas de las universidades del mundo; y para la mayoría de los profesores y estudiantes es una forma aceptada y hasta cierto punto exitosa de llevar a cabo la educación matemática universitaria.

Sin embargo, este enfoque presenta muchos problemas que son parte de las preocupaciones de la investigación en educación matemática a nivel universitario. En una revisión de la literatura, Abdulwahed, Jaworski y Crawford (2012) dan cuenta de los focos de atención de tal investigación, en particular cuando se trata de la investigación que propone innovaciones pedagógicas en contextos de ciencias, tecnología, ingeniería y matemáticas (CTIM, o STEM, en inglés):

- La importancia del cambio hacia enfoques de aprendizaje más centrados en el estudiante.

- Los problemas de la contextualización y del uso de situaciones reales como estrategia para facilitar las aplicaciones de las matemáticas.

- La necesidad de llenar vacíos en el aprendizaje previo de los estudiantes.

- La promoción del diálogo e intercambio entre estudiantes para la formación de discurso matemático.

- El mejoramiento de la motivación, interés y auto-eficacia de los estudiantes en el aprendizaje de las matemáticas.

Todos estos aspectos, concluyen los autores, se han discutido en proyectos cuya principal base han sido las teorías constructivistas del aprendizaje, con un foco en los individuos y en su construcción del conocimiento y competencias matemáticas. En este artículo es nuestra intención profundizar en lo que significa la conexión entre el formalismo matemático que los estudiantes universitarios aprenden, y sus posibilidades de recontextualizar las matemáticas dentro de un campo problémico y profesional dado. Tal discusión la haremos desde una perspectiva teórica socio-cultural, y utilizando las ideas del Problem Based Learning (PBL) o, en español, Aprendizaje Basado en Problemas (ABP) como se practica en el modelo pedagógico de la Universidad de Aalborg en Dinamarca. De esta forma contribuimos al campo de investigación de la pedagogía uni- 
versitaria de las matemáticas presentado un estudio conceptual basado en el análisis del trabajo de los estudiantes en el año inicial de ingeniería. Nuestro foco es mostrar cómo es posible organizar una práctica pedagógica que vaya más allá de las posibilidades y limitaciones que se encuentran documentadas en la literatura existente en el área.

\section{De la transferencia a la recontextualización y ensamblaje funcional}

Los estudiantes que están aprendiendo matemáticas como parte de su educación para la ingeniería, la tecnología o las ciencias naturales, necesitarán competencias especiales para conectar el formalismo abstracto propio de las matemáticas con un contexto de conocimiento diferente. Establecer una relación entre las formas de conocimiento matemático abstracto y otras áreas de conocimiento puede ser extremadamente difícil, si no a veces imposible. Esto resulta frecuentemente en una brecha entre, por un lado, la aparente competencia de los estudiantes universitarios para dar cuenta de los contenidos de los cursos de matemáticas, y, por otro lado, su falta de competencia para usar o aplicar matemáticas en otros campos de conocimiento. Incluso puede darse el caso de que estas dos competencias no se encuentren; es decir que independientemente de cuántas matemáticas abstractas avanzadas se le enseñen a los estudiantes y ellos efectivamente demuestren haberlas aprendido, ello no necesariamente contribuye a cerrar esta brecha.

Este tipo de problemática se ha llamado de diferentes maneras: el problema de la transferencia, la aplicación o la contextualización de las matemáticas. El problema de la transferencia de competencias desde un ambiente de matemáticas formales a un ambiente de matemáticas aplicadas ha sido estudiado por la investigación educativa en ciencias y matemáticas a nivel universi-tario (e.g., Bingolbali y Monaghan, 2008), y se ha discutido ampliamente desde las teorías socio-culturales del aprendizaje y conocimiento (e.g., Beach, 1999; Lobato, 2006). Basado en las teorías del aprendizaje situado y el avance de teorías socio-culturales del conocimiento para el estudio de la educación científica y matemática, Roth (2008) ofrece evidencia de que el supuesto de la tras-ferencia de conocimientos de un área y de un contexto a otro no es sostenible como un principio para la enseñanza de las matemáticas en la universidad. Al estudiar sistemáticamente y en muchos contextos diferentes la manera como distintos expertos desarrollan una competencia matemática general como lo es la lectura de gráficas, Roth presenta evidencia fuerte de que cualquier tipo de persona -desde el profesor titular de matemáticas, hasta el estudiante, pasando por personas en ámbitos de trabajo- desarrolla habilidades específicas de lectura de gráficas con relación al tipo de gráficas y el tema con el que suele trabajar. Estos hallazgos sugieren que la incapacidad de trans-ferir de los estudiantes del curso de matemáticas a otras áreas como la física o las ingenierías no son un problema intrínseco a las capacidades de conocimiento de los estudiantes, sino más bien un malentendido del sistema de enseñanza y aprendizaje sobre cómo funciona el pensamiento humano y en qué debería consistir el aprendizaje de las matemáticas y las ciencias y las competen-cias asociadas con ellas. Para abordar este problema en la enseñanza universitaria, Roth sugiere el uso de pedagogías activas como el aprendizaje basado en problemas (ABP) como una forma que puede abrir posibilidades de generación de conocimiento con relación a diversos contextos. 
Alineamos nuestra posición con la de Roth. Por eso nótese que aquí no nos referimos a esta brecha en términos de transferencia, ni tampoco de formalismo ni de aplicaciones. Dentro de la perspectiva teórica socio-cultural a la que nos adherimos para estudiar los procesos de aprendizaje en ambientes interdisciplinares no tiene sentido hablar de aplicaciones pues este término señalaría que un conocimiento o una competencia puede simplemente ajustarse, lo cual implicaría que es posible hacer una transferencia de conocimientos y habilidades de un contexto de conocimiento a otro. En cambio decidimos usar el término uso. Con inspiración en Wittgenstein (1997), para nosotros el uso se refiere al hecho de que cada tipo de conocimiento y de competencia son parte de un juego de lenguaje asociado con prácticas y reglas determinadas, y desarrollado en ámbitos contextuales definidos. El término recontextualización indica que cada vez que una persona entra en un campo específico y nuevo de conocimiento y práctica, la persona se involucra en un proceso complejo de reconstrucción contextual dentro de un juego de lenguaje diferente, pero que guarda similitudes con aquellos ámbitos y juegos que ya conoce. Más precisamente, un uso recontextualizado de cualquier conocimiento surge de la actividad de tomar aquellos recursos disponibles -bien sea conocimientos, destrezas o habilidades de un campo o de varios, o también de artefactos materiales como laboratorios, el internet o una computadora de mano- $y$ articularlos de una manera tal que su operación en un contexto determinado permitan abordar los problemas o retos que demandan la situación de una manera efectiva. Esta actividad es a la que llamamos ensamblaje de recursos que es posible usar para actuar adecuadamente en una situación. El ensamblaje es mucho más que la suma de cada uno de sus componentes ya que, si fuese posible desarticular cada uno de los recursos y habilidades que una o varias personas trajeron a esa construcción, ninguna de las partes podría por si misma poder atender a los requisitos de actuación apropiada en la situación. Además, ser parte del ensamblaje cambia también cualitativamente a cada uno de los componentes y también a los participantes del ensamblaje. En otras palabras, ninguno de sus elementos -humanos o no humanos- pueden funcionar el uno sin el otro. Es su conjugación y articulación lo que les da un sentido y función. La relación entre ellos es lo que se convierte en lo importante para el propósito de su uso.

Aquí es necesario un pequeño ejemplo. Dentro de las visiones de las aplicaciones de los conocimientos abstractos de las matemáticas, un punto importante es la capacidad de la persona para saber qué tipo de conocimiento se requiere en un campo de aplicación determinado. El típico ejemplo que cualquier estudiante universitario se ha encontrado es un modelo de movimiento parabólico con funciones de segundo grado. En este caso el movimiento y la función se pueden poner juntos en un modelo; pero se supone que la función es un objeto matemático que no cambia por el hecho de ser parte de un modelo de movimiento. Dicho de otra manera, la idea de la invariabilidad y la existencia independiente de cualquier objeto matemático del contexto dentro del cual se puede aplicar es la esencia misma del supuesto de abstracción y, por lo tanto, del poder de las matemáticas como la colección de objetos abstractos -descontextualizados- que pueden vestirse con las distintas ropas de cada contexto de juego en el que sea necesario y apropiado ponerlos.

Lo que proponemos con la idea del ensamblaje es el esfuerzo por dejar a un lado este supuesto para reconocer que los distintos recursos y herramientas que se articulan en un ensamblaje de uso cambian al ser parte de tal ensamblaje. Algunos de los conceptos de Latour (1994) para dar 
cuenta de la mediación tecnológica son de inspiración para entender de una manera diferente lo que significa poner en juego los recursos de las matemáticas en un ambiente interdisciplinar como la que se promueve en las formas de trabajo del aprendizaje basado en problemas (ABP). En proceso de mediación tecnológica ninguno de los componentes, ni humanos ni no-humanos, pueden entenderse de manera aislada. Sus relaciones son aquello que les otorga características y posibilidades de acción. Esta idea es fundamental para permitir un desplazamiento de nuestra concepción sobre la esencia de los conocimientos matemáticos en una situación donde entran en contacto y necesitan ponerse en juego dentro de un contexto diferente al de las matemáticas mismas. Desde el punto de la acción científica tecnológica, el poder de las matemáticas no está en lo que ellas de por si son como objetos de pensamiento abstracto, sino más bien en las conexiones y modificaciones que pueden surgir para ser parte de ensamblajes de acción. Más adelante, y con el análisis que presentaremos, explicaremos esta idea. Por ahora, es suficiente decir que, desde nuestra perspectiva, el foco de los procesos de aprendizaje de los estudiantes están en cómo aquello que reconocemos como herramientas y recursos matemáticos hacen partes de ensamblajes funcionales dentro de los problemas tecnológicos a los que los estudiantes se enfrentan.

También hablamos de competencias para señalar el hecho de que todo conocimiento está presente sólo en relación con una acción. Para nosotros no es posible hablar de destrezas o habilidades como capacidades independientes de la participación y acción en prácticas de generación de conocimiento y de aprendizaje. En este sentido, competencias, destrezas y habilidades son inseparables en la acción. Lo que las hace activarse es su relación cuando alguien hace algo con otros y con artefactos para lograr un propósito. De nuevo, competencias, destrezas y habilidades surgen y se activan en un ensamblaje de actividad.

En este artículo analizamos el enfoque pedagógico del ABP. No investigaremos el modelo como un modelo teórico de aprendizaje entre otros, ni compararemos diferentes tipos de ABP. Esto ya se ha hecho anteriormente en la literatura (e.g., Barrows, 1986; Niss, 2002). Más bien, nos enfocaremos en analizar tres casos ejemplares provenientes de experiencias con estudiantes del ciclo básico de ingeniería y ciencias de la Universidad de Aalborg, para mostrar cómo los estudiantes recontextualizan y ensamblan las herramientas y recursos matemáticos con otros tipos de conocimientos para generar comprensiones funcionales y apropiadas en los contextos dados.

Para comenzar, presentaremos algunos de los elementos clave del modelo ABP en la Facultad de Ingeniería y Ciencias de la Universidad de Aalborg. A continuación analizaremos dos casos de proyectos realizados por dos grupos de estudiantes. En base a los casos, discutiremos las características de su actividad matemática, en especial en lo relacionado con el cierre de la brecha entre formalismo y uso. Concluimos con unas reflexiones sobre las ventajas de este tipo de ambientes de enseñanza universitaria.

\section{Breve reseña del ABP en Aalborg}

La Universidad de Aalborg se creó a comienzos de la década de 1970 y se desarrolló en el espíritu de cambio que marcaba esa época de revolución estudiantil en Europa. Un aspecto de estos cambios fue la atención que se prestaba al espacio y procesos de aprendizaje que ocurrían en las 
universidades. La educación universitaria estaba acusada de tratar sólo con teoría abstracta en la «torre de marfil» de la academia, en lugar de enfocarse en problemas del mundo real que ocurrían fuera de los muros de la universidad (Illeris, 1974). En un proceso histórico complejo donde casi toda la retórica política inicial ha desaparecido gradualmente -o por lo menos ha sido transformada radicalmente- el modelo ABP de Aalborg es, en la actualidad, principalmente y ante todo, un sistema educativo eficiente que satisface las demandas tanto del gobierno para cumplir con metas de eficiencia educativa, como de los empleadores con respecto a las cualificaciones y competencias de los estudiantes graduados de la universidad. Este sistema es de hecho una diversidad de modelos educativos específicos en las diferentes facultades y departamentos de la Universidad, y por ello puede ser difícil señalar una característica central única del modelo ABP de Aalborg. No obstante, en lo que sigue, trataremos de describir algunas de las características fundamentales sobre las cuales tratamos de construir especificidades adaptadas a diversos programas de estudio. Para un recuento más elaborado y profundo del modelo ABP de Aalborg, véase Kolmos, Fink y Krogh (2004), Kolmos (2008), y Krogh y Jensen (2013), y también http://www.en.aau.dk/aboutaau/aalborg-model-problem-based-learning/.

El ABP no sólo está basado en la definición de problemas que guían el proceso de aprendizaje, sino que también se organiza en proyectos colectivos realizados por un grupo de estudiantes de hasta ocho individuos. Este número varía mucho y normalmente decrece de manera gradual a medida que los estudiantes se especializan y tienen más experiencia. Cada grupo tiene que involucrarse en el proceso de indagación para abordar un problema, bien sea práctico o teórico, definido por ellos y que resulta en la producción semestral de un reporte de proyecto, en la mayor parte de los casos con una extensión de entre setenta a ochenta páginas. En el primer año de ingeniería y ciencias cada grupo está asociado con dos facilitadores para el desenvolvimiento del proyecto. Si tomamos, por ejemplo, un grupo de científicos de la computación, uno de los facilitadores es un experto en ciencias de la computación, normalmente un miembro del cuerpo profesoral del departamento de ciencias de la computación. Este es el facilitador principal y es el responsable de apoyar el avance del grupo en las competencias técnicas de algún área de las ciencias de la computación. El otro facilitador es responsable de la contextualización del contenido técnico pertinente. Este segundo facilitador juega un papel importante en apoyar la investigación de los estudiantes ofreciendo perspectivas de contextualización. Esta persona es normalmente miembro del personal académico de otra área afín. En este caso, Ole Ravn ha sido el facilitador contextual de los proyectos que examinaremos.

Además de la escritura del informe del proyecto, los estudiantes también asisten a diversos cursos magistrales. Durante el primer año de estudios, algunos cursos ayudan a construir las competencias de trabajo colaborativo requeridas para funcionar en un entorno propio para el modelo ABP -el foco está en los procesos de aprendizaje en los grupos, tal como la cooperación en equipos o el compartir conocimiento, entre otros. Otros cursos magistrales tradicionales apoyan los perfiles disciplinares de los estudiantes y aportan las herramientas básicas en varias de las disciplinas fundamentales para las ingenierías. Todos los ingenieros reciben cursos matemáticos extensos en los temas tradicionales típicos del primer ciclo universitario y, además, cada rama de ingeniería tiene cursos de apoyo en sus disciplinas particulares. El balance entre las actividades 
de los cursos y el trabajo de grupo para el proyecto se inclina, sin embargo, hacia éste último. De los treinta puntos ECTS (European Credit Transfer System) que definen un semestre completo de estudios, al menos quince se adquieren por razón del trabajo en el proyecto. Este número puede ser más alto en algunos programas de ingeniería y ciencia. En otras palabras, la mitad o más de la carga académica de un estudiante durante un semestre corresponde a su trabajo en el proyecto con el grupo de estudiantes al que pertenece.

Además, la organización en el tiempo de un semestre del estudio de cursos y del trabajo en proyectos tiende a ser tal que, al inicio del semestre hay más actividad de asistencia a cursos, mientras que el trabajo en los proyectos colectivos apenas se inicia. A medida que transcurre el tiempo, el tiempo dedicado a cursos disminuye mientras que la actividad de trabajo en los proyectos aumenta, hasta tal punto que, más o menos un mes antes de terminar el semestre, los estudiantes se dedican casi de tiempo completo a trabajar en sus proyectos. Esto también significa que los profesores, hacia el inicio del semestre, tienen la función de dar clases; y a medida que el semestre avanza cambian su papel a actuar como facilitadores de los grupos.

Con respecto a la evaluación, hay cursos que tienen su propio examen y el sistema de asignación de calificaciones para cada curso puede ser con una escala numérica, o simplemente con un «aprobado» o «no aprobado». Sin embargo, el gran examen de cada semestre está asociado con la presentación y defensa del informe del proyecto en exámenes grupales (e.g., Dahl y Kolmos, 2015). Estas sesiones de exámenes grupales no sólo buscan que los estudiantes muestren que todos y cada uno de ellos manejan todo el proceso y contenido del trabajo del grupo y su resultado, plasmado en el informe del proyecto. También se busca evaluar a través del proyecto los objetivos generales del semestre e incluso lo que se ha aprendido en los otros cursos de apoyo que los estudiantes cursaron. En este sentido, la evaluación de un proyecto se convierte en una situación donde se espera que los estudiantes puedan hablar con propiedad de lo que aprendieron en el proyecto y en el semestre.

Un ingrediente importante en el sistema educativo del modelo $A B P$ es el entorno físico. Los grupos de trabajo requieren salones adecuados y, por tanto, la Universidad ha invertido una extensión considerable de su planta física en construir espacios de oficina para asegurar que cada grupo de estudiantes tenga un salón propio a su disposición. Esto proporciona a los estudiantes la oportunidad de encontrarse cada día en su propia «oficina» con su propio refrigerador, tableros, computadores portátiles y demás elementos, para crear un ambiente y un espacio de trabajo que supla bien sus necesidades. Esta parte del sistema educativo no se implementa de igual manera en todos los campos universitarios, pero en las facultades de ingeniería y ciencias y también de medicina este sistema está totalmente desarrollado; mientras que en la facultad de humanidades el espacio físico se administra de manera diferente.

En lo que sigue, describiremos la organización general de los estudios en el primer año de ingeniería y ciencias en la Universidad de Aalborg. Con esto tenemos la intención de mostrar cómo es posible crear un ambiente para el aprendizaje y enseñanza de las matemáticas a través del modelo $\mathrm{ABP}$ en todas las posibles ramas de ingeniería. 


\section{El modelo ABP en acción}

Los estudiantes cuyos proyectos describiremos a continuación deben trabajar en un tema general que han de elegir de un abanico de temas formulados por el grupo de facilitadores. Por ejemplo, en años recientes los estudiantes han podido elegir de un conjunto de temas como: técnicas de microarreglos de ADN para apoyar el diagnóstico de enfermedades, el sistema Pagerank de Google, escenarios de propagación la gripe aviaria, entre otros. Para cada uno de estos temas -a menudo, aproximadamente diez temas para un semestre dado- el grupo de facilitadores ha desarrollado una descripción de una página de extensión donde se plantean aspectos importantes de posible problematización con respecto a esos temas. Estos están por lo general relacionados con los temas de investigación del facilitador -que es profesor investigador de la universidad. Este marco de referencia sirve a los estudiantes para poderse imaginar y posteriormente definir un posible proyecto. Cuando los estudiantes eligen un tema dado, comienzan a negociar la definición de un problema abierto de indagación en ese tema, sobre el cual han de escribir un informe a lo largo del semestre. En algunos pocos casos, los estudiantes aportan ideas con respecto a temas nuevos sobre los cuales escribir, y éstos son bien recibidos por los facilitadores si el área de interés presenta problemas matemáticos o técnicos potencialmente fructíferos.

Después de que un grupo de estudiantes elige un tema, se inicia un proceso de investigación colectiva en el grupo. El grupo de estudiantes se involucra en un proceso arduo de exploración del tema con el objetivo de definir un problema que guiará el proceso de aprendizaje. Un problema en este contexto no es un ejercicio ni una situación problémica en el sentido típico matemático o de las ciencias. Así, el significado de «aprendizaje basado en problemas» difiere de pedagogías como el aprendizaje problémico o la resolución de problemas como se conoce en el ámbito de la investigación en educación matemática (e.g., Schoenfeld, 1992). Vithal, Christensen y Skovsmose (1995) analizaron hace un tiempo este enfoque; más recientemente Hernández, Ravn y Valero (2015) lo caracterizaron desde una perspectiva teórica socio-cultural.

Un problema se refiere a una situación abierta que genera un reto de conocimiento y que demanda una solución teórica y/o práctica que permita abordarla. El problema puede tener un anclaje en la realidad social o tecnológica o en un ámbito teórico. Independientemente de esto, el problema siempre es un problema «real» en el sentido de que es de interés y de importancia no sólo para los estudiantes sino para otros, bien sea una comunidad disciplinar, una comunidad profesional, unos usuarios, o la sociedad en general. Esto quiere decir que el problema no es algo que «pone el profesor» o que se «saca de un libro» sino que es una construcción que hacen los estudiantes a partir de sus intereses y con respecto a lo que se considera relevante en la esa realidad. Además, el abordaje del problema requiere un proceso de investigación colectivo que se extiende por un semestre. No estamos hablando de un problema que se resuelve en unas pocas horas o en una semana. En contraste con la variedad de significados del concepto de problema como aquello que guía la actividad de aprendizaje de los estudiantes, el significado de éste en el modelo de la Universidad de Aalborg se relaciona con una organización curricular que lo hace asemejar a un proceso investigativo abierto, colectivo y guiado por expertos en el campo de conocimiento del temático del problema. 
En el proceso de abordar problemas a través de proyectos, el grupo trabaja en informes de avance sobre su trabajo. Estos informes son discutidos con dos profesores que toman el papel de facilitadores del proceso de los estudiantes. Con frecuencia, el proceso de investigación y aprendizaje es no lineal; es muchas veces frustrante y gira en torno de asuntos interdisciplinarios: ¿Cuál es el problema general que se quiere abordar? ¿Cómo se puede formular claramente este problema? ¿Qué clase de matemáticas formarán parte central de este informe del proyecto? ¿Deberíamos buscar información empírica para trabajar sobre ella? ¿Necesitaremos crear un nuevo algoritmo o incluso implementar un uso de la teoría desarrollada por nosotros mismos para resolver nuestro problema? ¿Qué tan detallado debería ser un recuento de las diferentes partes de la teoría usada? Y la lista de preguntas continúa y continúa. Los estudiantes elaboran una estrategia para completar un informe del proyecto de manera que en ella se haga explícitos los elementos de diversas teorías utilizadas, entre ellas la teoría matemática que sustenta las nociones y procedimientos escogidos por el grupo. El grupo también tiene que dar cuenta de cómo los conocimientos seleccionados se convierten en herramientas útiles en un contexto dado. Esto se lleva a cabo en cooperación estrecha con ambos facilitadores. Como resultado del proceso, los estudiantes escriben en el informe textos propios donde integran distintas fuentes para dar cuenta de las matemáticas de las que necesitan apropiarse y también de la contextualización que hicieron de ellas en el proceso de ponerlas en uso en un contexto determinado.

A continuación entramos en el detalle de dos casos de proyectos de grupos ABP reales realizados por estudiantes de primer año. Al utilizar dos casos como base empírica para nuestra discusión, nos apoyamos en varias ideas metodológicas importantes. Primero, decidimos alejarnos de la idea de que la validez de nuestras reflexiones se encuentra en una representatividad estadística del trabajo de los estudiantes. Nuestra intención no es generalizar lo que sucede con los estudiantes de primer año en la Facultad de Ingeniería y Ciencias de la Universidad de Aalborg cuando trabajan con el ABP. Nuestra estrategia está más en un estudio cualitativo minucioso de las producciones escritas de dos grupos de estudiantes para poder generar nuevas posibilidades de pensamiento sobre aquello que parece habitual o incluso generalizado. Este tipo de enfoque se ha discutido en la educación matemática como principio de la investigación crítica (e.g., Vithal y Valero, 2003: 578-580). Knijnik (2012), apoyada en Wittgenstein (1997), discute cómo el ejemplo puede cumplir un papel en iluminar una situación que va más allá de ser una simple ilustración. El «poder del ejemplo» como estrategia para generar nuevas interpretaciones (Flyvbjerg, 2001) es el tipo de principio al que nos acogemos. Cada uno de los dos casos que examinaremos ilustra diferentes logros que los estudiantes han tenido a través del modelo ABP. Adicionalmente, los casos muestran cómo los estudiantes, dependiendo de la asignatura específica, son guiados por los facilitadores e impulsados por la dinámica de su propio grupo para generar nuevos ensamblajes de conocimiento.

\subsection{Escenarios de propagación de la gripe aviaria}

Hace unos pocos años, la aterradora gripe aviaria explotó con riesgo de convertirse en una pandemia. En todas las noticias a nivel global se escuchaba sobre casos de contagio, y hubo varios informes alarmantes de la amenaza de propagación de la enfermedad en el territorio danés. En cuestión 
de unos pocos y angustiosos días, como resultado de un amplio cubrimiento de la prensa sobre la propagación de la gripe aviaria, se vendieron en todo el mundo cantidades grandes de píldoras de Tamiflú, una medicina antiviral para el tratamiento de la gripe. Se tomaron medidas políticas tanto a escala nacional como global para contener el problema.

Un grupo de estudiantes eligió como proyecto examinar escenarios de propagación de tal enfermedad. El grupo, conformado por cinco estudiantes de primer semestre y registrados en el programa de matemáticas y ciencias de la salud (sundhedsmatematik, en danés), tenía un facilitador para los aspectos contextuales y un facilitador para las matemáticas, como se mencionó antes. El primero dedicaba un tercio del total de sus horas de supervisión a este grupo en particular. Los estudiantes decidieron enfocarse en un escenario danés de propagación. Si la enfermedad llegara a alcanzar las proporciones de la pandemia de la Gripe de 1918, tal y como los medios de comunicación sugerían, los efectos serían devastadores. El grupo decidió investigar:

¿Han provocado los medios de comunicación una reacción exagerada en los políticos y en la población frente al riesgo mundial de la gripe aviaria, y se puede decir que hay un riesgo en Dinamarca? (VVAA, 2005: 22)

Modelar la propagación de la gripe aviaria se podría enfocar de muchas maneras. Asimismo, saber cómo elaborar el modelo apropiado de una situación dada es naturalmente parte de lo que significa tener competencia en ensamblar las herramientas matemáticas en un modelo de propagación de un virus. Pero, ¿cómo hacer el modelo matemático sin más conocimiento del fenómeno en cuestión? Para abordar el problema, el grupo decidió articular tres perspectivas científicas, cada una de las cuales fueron examinadas: la biomédica, donde se dio cuenta de lo que es un virus y su reproducción, al igual que las formas de contener el contagio; la política, donde el grupo analizó cómo el sistema político globalmente y en Dinamarca había tratado de hacer frente al riesgo de pandemia; y la sociología de los medios de comunicación, donde se abordó el tema de cómo los medios influyen en su audiencia, y cómo en este caso particular los medios daneses habían presentado la información sobre la gripe aviaria al público. Para esto, los estudiantes buscaron diferentes fuentes y lograron escribir un texto de 15 páginas como análisis de su problema. En el texto exponen los conceptos biológicos relacionados con la gripe aviaria: ¿qué es y qué tan contagiosa es?, ¿cómo se transmite entre los seres humanos?, ¿cuál es la tasa esperada de éxito del antídoto Tamiflú contra una pandemia?, etc. También dan cuenta de cómo la población, los políticos y los medios se influyen unos a otros y cómo se dio tal mutua influencia en el caso del riesgo de la pandemia de gripe aviaria en Dinamarca.

Estas investigaciones acerca del escenario cambiaron el conocimiento del grupo y por tanto su perspectiva acerca de lo que diversas herramientas de modelación matemática podrían hacer para generar un modelo de propagación de la gripe. El objetivo del proyecto fue entonces elaborar un modelo del desarrollo de la gripe aviaria en una epidemia o una pandemia, en caso de que se diera una mutación del virus H5N1 que lo hiciese contagioso entre seres humanos. Por esta razón, los estudiantes determinaron que tenían que trabajar con la modelación de situaciones de crecimiento. Esta podría hacerse con un modelo estocástico o con uno determinista; o también un modelo continuo o discreto. Después de sopesar las alternativas que cada uno de estos tipos de modelo ofrecen, los estudiantes decidieron: 
escoger un modelo determinista porque sería lo más fácil de construir, pues sería más manejable variar y evaluar cada uno de los parámetros, que si trabajáramos con un modelo estocástico (íbid: 27).

Además, el modelo involucraría ecuaciones diferenciales pues las funciones de cambio, donde sea posible tener cocientes diferenciales, parecían apropiadas para cálculos de propagación en un escenario dado. Por esto, el modelo también debería ser continuo. Los criterios en general para el modelo determinista que los estudiantes fijaron son:

1. Debe modelar una parte de la realidad para así poder fácilmente evaluarla; 2. Debe describir cambio incremental; 3. Debe haber correspondencia entre el modelo y la realidad (íbid: 27).

El proceso de construcción del modelo está documentado en los tres capítulos centrales del informe del proyecto (íbid: 28-64). Con base en distintas fuentes, los estudiantes escribieron un texto sobre lo que son las ecuaciones diferenciales y por qué son apropiadas para abordar problemas de crecimiento y cambio. Sus explicaciones siempre estaban relacionadas con el escenario de la propagación de un virus:

El crecimiento es algo que cambia. Si no hay un límite en esta magnitud, puede crecer hasta el infinito. En este proyecto trabajamos en un conjunto acotado, es decir, nunca puede haber más personas infectadas que el total de individuos en la población. Por lo tanto debe haber un límite de qué tan grande el crecimiento puede ser, con respecto a qué tan grande es el conjunto acotado. Por eso tomaremos una función de crecimiento con un límite natural. Esto podría ser la ecuación logística (íbid: 30).

Teniendo en cuenta estas consideraciones, comenzaron formulando un modelo muy simple de contagio de una enfermedad en condiciones donde todos los individuos de una población potencialmente podrían contagiarse. Sin embargo, este no es un modelo realista para este caso, pues se conoce que, en caso de epidemias, no toda la población se comporta homogéneamente. El modelo «SIR» (Renshaw, 1991), que considera cambios en la población entre Susceptibles, Infectados y Recobrados, tuvo que incluirse como parte de las consideraciones, pues un modelo más acertado debería poder determinar las clases en las que la población se divide de acuerdo con su riesgo de infección. Esto tiene implicaciones importantes para poder acotar tanto la población como la propagación de un virus. Además, consideraciones sobre el periodo de infección y la rata de infección deberían ser parte del modelo. Pero esto no era suficiente. Dado que el interés del grupo era «saber si es posible que la enfermedad [gripe aviaria] se desarrolle en una epidemia, es necesario investigar qué criterios deben cumplirse» (íbid: 43) para que este fuera el caso. En el modelo esto significaba pensar en el valor límite de riesgo de contagio de la enfermedad con respecto a las distintas variables involucradas.

El modelo que los estudiantes construyeron, formado por sistema diferencial compuesto por tres ecuaciones diferenciales, fue evaluado utilizando distintos métodos y variando algunos de los supuestos teniendo en cuenta lo que ellos significan en la realidad. Por ejemplo, es plausible suponer que toda la población de hecho puede entrar en la clase I (infectados)? ¿Y qué pasaría si hay medidas de aislamiento para evitar que ciertas personas en la clase $S$ (susceptibles) no terminen en I (infectados)? ¿Y si se tiene en cuenta que hay muertes, el tamaño de la población cambia en el tiempo? Además, cada tipo de virus tiene determinados comportamientos de latencia y de 
mutación. La conclusión después de probar el modelo en distintas simulaciones y razonar sobre las limitaciones de cada una -incluyendo simulaciones de escenarios «realistas» e incluso una prueba de «escenario de peor caso»- fue que era improbable que la gripe aviaria se desarrollara en un virus con potencial de generar una epidemia, entre otras porque muchas de las iniciativas internacionales de prevención, según las simulaciones en el modelo, podían tener efecto. Por eso, las iniciativas políticas no están de menos, así no haya riesgo de epidemia. La gripe aviaria es un riesgo para Dinamarca, entendido como que debemos estar alertas, pero sus efectos no serían devastadores, pues es casi impensable que una epidemia se desate. El problema es si los medios han provocado una reacción exagerada en la población y en los políticos (íbid: 71).

El análisis interdisciplinario del problema contextual con relación al sistema de ecuaciones diferenciales que el grupo construyó le permitió a los miembros del grupo enfrentarse a más que la mera solución exacta (o numérica) a una ecuación diferencial. Aprendieron sobre asuntos de confiabilidad con respecto a los modelos matemáticos, y sobre cómo las diferentes constantes en las ecuaciones matemáticas afectan considerablemente los resultados de investigación en un ámbito complejo de uso.

De otra parte, estos estudiantes abordaron un fenómeno que no se puede interpretar en términos matemáticos sin las consideraciones de otras perspectivas científicas: de ahí su enfoque interdisciplinario al problema. La mayoría de los problemas de la vida real, de la ciencia y la ingeniería se parecen al que ellos estudiaron en que su complejidad involucra una cantidad de dimensiones que requieren conocimientos y enfoques de resolución de problemas en varias ramas de la ciencia. Saber algo de los tipos de conocimiento que otras ciencias son capaces de manejar también hace posible que los estudiantes de matemáticas lleguen a ser conscientes de cómo las competencias matemáticas específicas marcan particularmente el trabajo de los científicos.

\subsection{El algoritmo Pagerank de Google}

En una sociedad donde crecientemente se usa el internet para la búsqueda de información, surge el problema de qué tan confiable es la información que se recibe dado que se sabe que, comparado con sistemas anteriores como las bibliotecas, el internet no tiene control sobre quién provee información ni tampoco sobre la veracidad de la información suministrada. Por eso, le queda al usuario el problema de juzgar, a partir del sentido común, si la información que recibe es confiable. Entender cómo los motores de búsqueda del internet funcionan y cómo producen una jerarquización de los resultados de una búsqueda fue el problema inicial de un grupo de ocho estudiantes de segundo semestre en el año de 2007. El grupo, conformado por 2 estudiantes de ciencias de la computación y 6 estudiantes de matemáticas, eligió trabajar con el algoritmo PageRank de Google pues en esta temática general podían

combinar matemáticas con ciencias de la computación evitando hacer una demarcación tajante entre las dos áreas (VV.AA, 2007: 1).

Las preguntas iniciales para el trabajo fueron: ¿Qué criterios de calidad debe cumplir un motor de búsqueda en el 2007? ¿Qué papel cumple Google como un motor de búsqueda del internet? 
En el proceso de delimitar su problema dentro de la temática, el grupo comenzó por describir cómo la idea de la búsqueda de información ha cambiado en el tiempo, para después definir lo que es un motor de búsqueda en el 2007. El papel del sistema de bibliotecas como grandes centros de información cambió radicalmente con el desarrollo del internet. A pesar de la facilidad del acceso a la información, el gran problema es la confiabilidad de la información dada la falta de control sobre quién y qué se sube a la red, y además, el hecho de que muchas compañías tienen intereses comerciales o políticos en promoverse al posicionarse arriba de la jerarquía de resultados de búsqueda de un motor. También surgió una «competencia entre distintas compañías de internet en hacer el motor que diera los mejores resultados». Por todas estas razones es importante entender qué hace un motor de búsqueda y qué criterios de calidad emplea para desplegar sus resultados.

Tras caracterizar y analizar diferentes tipos de motor de búsqueda -booleano, vectorial, probabilístico y combinado-, se propusieron identificar los elementos que lo componen, pues algunos de los tipos de búsqueda anteriores no necesariamente se limitan al internet. Por ejemplo, algunos de ellos se usan en sistemas cerrados de bases de datos en bibliotecas. Tras identificar los elementos, el grupo se propuso definir los criterios de calidad de la jerarquización de resultados. Con base en sus propias reflexiones y en el artículo de Xiaolan Zhu y Susan Gauch, «Incorporating quality metrics in centralized/distributed information retrieval on the World Wide web» en las memorias de la «23a Annual International Conference of the Association for Computing Machinery's Special Interest Group on Information Retrieval (SIGIR)» (http://sigir.org/), los estudiantes propusieron cinco parámetros de calidad: amabilidad del diseño para el usuario, rapidez de los resultados, precisión de los resultados, calidad de los resultados y credibilidad. Con estos criterios en mente, el grupo comenzó a analizar Google como compañía que muy rápidamente se ha expandido y cuyo buscador es utilizado masivamente. De los muchos caminos posibles a seguir, se concentraron en la calidad de los resultados de una búsqueda pues este es uno de los factores que han hecho a Google tan popular. El problema inicial definido se delimitó en dos nuevas preguntas a indagar: ¿Cuál es la base matemática del algoritmo PageRank de Google? ¿En qué grado cumple este sistema con los criterios de calidad de la búsqueda de resultados?

Como el grupo estaba conformado por futuros matemáticos y futuros científicos de la computación, se dedicó mucho tiempo del proyecto y espacio del informe a ganar conocimiento y presentar información sobre la estructura del Internet cuando se le considera como un grafo. Esto incluyó teoría básica y avanzada de grafos, lo mismo que el conocimiento sobre el uso de álgebra lineal básica, vectores y matrices (¡especialmente la enorme matriz Google!). Además, el informe del proyecto trató tangencialmente el uso de matrices estocásticas y cadenas de Markov para entender el WWW como un gran grafo. Finalmente, se analizó el aparato matemático que sustenta el PageRank de Google y se analizó con referencia a las cinco criterios de calidad desarrollados al comienzo del informe.

Del análisis surgieron diversas críticas tanto a los procedimientos como a la jerarquización de información que Google le presenta al usuario. El grupo se detuvo en los dilemas éticos sobre los criterios menos obvios utilizados por el algoritmo PageRank de Google y se analizó de qué manera la organización matemática del sistema tenía algunos beneficios pero también algunas deficiencias 
desde el punto de vista de los usuarios. Dada la importancia de la jerarquía en la presentación de la información, el problema de la optimización de los motores de búsqueda ha surgido:

El concepto optimización de motores de búsqueda cubre tanto intentos éticos como no éticos para darle a una página un puesto tan alto en la jerarquía como sea posible, de tal manera que aparezca como el primer resultado en la lista de la búsqueda. Dado que las páginas web de compañías y organizaciones [...] son un instrumento muy importante para vender productos o servicios, informar sobre nuevas iniciativas y también por supuesto ganar nuevos clientes, ha surgido toda una nueva rama que se especializa en la optimización de motores de búsqueda, los llamados SEOs (search engine optimizers) (íbid: 74).

Los estudiantes discutieron lo que es el comportamiento ético y no ético de un SEO:

El SEO ético es un profesional en la construcción de páginas web, que usa todas las finezas técnicas para presentar el contenido, diseño y graficos de la mejor manera. Se enfoca en mantener y actualizar las páginas de tal manera que aparezcan tan efectivas y atractivas como sea posible. En su comunicación con el dueño de la página le informaría que si se utilizan otros métodos no éticos, como algunas formas de spamming, se corre el riesgo de obtener un castigo por parte del motor de búsqueda [...] Esto no sucede con el SEO no ético. Este utiliza todos los métodos ilegales para mejorar la posición de una página en la jerarquía de búsqueda, y por lo tanto es uno de los enemigos del motor de búsqueda (íbid: 74).

La discusión de los métodos no éticos para alterar la posición en una búsqueda -como son el spamming con enlaces y las bombas Google- llevó a los estudiantes a discutir aspectos como la mala propaganda que se le puede llegar a crear a una organización o una compañía con el uso de estos métodos. En otras palabras, puede haber una cantidad de manipulación con la información y jerarquía de las búsquedas que presenta Google, y el sistema de PageRank no es inmune a ella.

La conclusión del análisis de los estudiantes apunta a que, de los criterios de calidad especificados, la popularidad medida en términos de un análisis de enlaces es un criterio importante para la calidad de las búsquedas. De hecho, este tipo de medición es la base del funcionamiento de PageRank en Google. A pesar de que el algoritmo que usa Google es secreto, los estudiantes pudieron analizar que:

Google usa con gran éxito el análisis de enlaces en su motor de búsqueda, lo cual lo ha hecho uno de los más usados motores hoy en día [...] La teoría de grafos se usa para modelar el internet, donde las páginas se representan con vértices y los enlaces se representan con las aristas entre los vértices. Se trata de que todas las páginas reciben un valor PageRank, que expresa que tan "importante" es la página, dependiendo de cuántas otras páginas se enlazan con ésta. Este valor se puede usar para jerarquizar los resultados de acuerdo con cuales páginas se presentan primero. El cálculo de matrices puede usarse para calcular los valores PageRank de todas las páginas en la red, a partir de construir en el grafo del internet una matriz de adyacencia que muestra la probabilidad de seguir un enlace de una pagina a la otra. Al encontrar un vector que cumple con la ecuación $A \vec{x}=\vec{x}$ se puede encontrar los valores finales donde $\vec{x}$ es el vectorPageRank, si los elementos son valores-PageRank de cada página. En el proyecto mostramos que tal vector existe y que puede calcularse con el método de la potencia (íbid: 87).

$\mathrm{Al}$ encontrar una forma de calcular este valor fue posible pensar en lo que significa la calidad de las búsquedas, cómo mejorar esa calidad y, por supuesto, cuáles son los riesgos de manipulación deliberada de la jerarquía. 
El grupo en cuestión mostró cómo un proyecto $\mathrm{ABP}$ podría integrar algo tan contemporáneo como la máquina de búsqueda de Google con un tema matemático, la teoría de grafos, que a menudo se ejemplifica con usos en problemas de reparto y matrices de probabilidad. El trabajo del proyecto de este grupo también señala el hecho de que una inmensa e impresionante cantidad de teoría - proveniente en parte de artículos y libros de investigación sobre el tema- se puede poner en juego en el modelo ABP, pues los estudiantes tuvieron que recurrir a estas fuentes de información para poder abordar su problema. Así, el modelo ABP ofrece la oportunidad de diferenciar entre los resultados de aprendizaje de diferentes grupos de estudiantes con relación a sus ambiciones, destrezas especiales, etc.

\section{El ABP y los ensamblajes matemáticos}

Después de presentar los casos anteriores, vamos a dirigirnos a una consideración más general del uso del modelo ABP de Aalborg con respecto al aprendizaje de las matemáticas de estos grupos de estudiantes. Es evidente que el modelo contrasta con la manera como se enseñan las matemáticas en muchos otros ámbitos educativos, y difiere, por ejemplo, de un curso tradicional en el que el profesor presenta a los estudiantes la teoría de alguna porción de las matemáticas y, luego, ellos trabajan con ejercicios o demostraciones de teoremas importantes. Esta forma dominante de enseñanza de las matemáticas entrena a los estudiantes en la sintaxis matemática pero, como se dijo al comienzo de este artículo, no garantiza necesariamente una comprensión significativa que sea la base para relacionar estas matemáticas con las características y procesos de un ámbito temático complejo en otro campo de conocimiento. Aludiendo a los casos, muchos de estos estudiantes de matemáticas se convertirán más tarde en profesores de matemáticas en diferentes niveles de educación, o trabajarán en áreas donde se requiera cierto tipo de indagación matemática en interacción amplia con otros profesionales de diferentes campos de estudio. En todas estas situaciones se requiere poder navegar en distintos ámbitos de práctica matemática dentro de una situación concreta y no sólo manejar matemáticas abstractas. Para los estudiantes de ingeniería, la posibilidad de manejar la brecha entre el formalismo matemático y el uso de herramientas matemáticas en otros contextos se podría considerar incluso más urgente, y esto podría sugerir la conveniencia de trabajar siguiendo alguna clase de modelo ABP al enseñar matemáticas a un grupo específico de estudiantes de ingeniería.

A partir de los casos se pueden destacar varios puntos acerca del modelo ABP como una estrategia pedagógica que permite el espacio para operar dentro de ambientes interdisciplinarios donde la creación de ensamblajes funcionales sea posible. En el primer caso, un grupo de estudiantes trabajó con ecuaciones diferenciales en un escenario altamente complicado con respecto a su nivel de estudios y conocimientos -recuérdese que este grupo era de primer semestre. El estudio del problema que terminaron formulando no se podría haber resuelto sin involucrarse profundamente en conocer la situación en donde las ecuaciones diferenciales iban a ser ensambladas con otros tipos de conocimientos de, por ejemplo, epidemiología. Este enfoque significó que los estudiantes aprendieran acerca de las diversas herramientas de conocimiento disponibles 
a medida que les daban sentido en el proceso de generar un ensamblaje funcional en el contexto del problema. Contrario a la idea que domina en muchos de los currículos de matemáticas universitarios donde la comprensión y manejo de la notación y el formalismo de las matemáticas se asume preceder a la posibilidad de un «uso», aquí se hace claro que el trabajo en es escenario del problema revierte y cuestiona esta visión curricular. Además los estudiantes aprendieron no solamente por qué una ecuación diferencial dada tiene esta solución exacta, sino que también aprendieron cómo deberían ser interpretadas las diferentes constantes en un escenario dado de aplicación y qué las habría hecho cambiar de valor, etc. Esto de nuevo les dio la oportunidad de reflexionar sobre la validez del modelo matemático porque, por ejemplo, si algún parámetro sobre el cual el modelo estaba construido era impreciso, ello podría significar una gran inexactitud para el modelo entero. Acercarse a comprender la estabilidad y elasticidad de estos modelos en primer semestre es un logro avanzado: este tipo de inexactitud podría tener consecuencias catastróficas en términos de la expansión de una enfermedad como la gripe aviaria.

Con el segundo caso, teníamos la intención de mostrar cuán impresionantes pueden ser algunas de las metas logradas por los estudiantes en el entorno de aprendizaje del modelo ABP. Incluso en grupos muy grandes, la capacidad para recoger información sobre una aplicación técnica tal como el motor de búsqueda de Google y el aparato conceptual y matemático que soporta esta aplicación técnica se puede desarrollar y aprender exitosamente. No es extraño que los grupos de estudiantes de primer año lean artículos de investigación que abordan específicamente el problema que ellos pretenden resolver. Con frecuencia, por supuesto no tienen todas las herramientas para comprender los detalles de tales artículos, pero con la ayuda de sus facilitadores y de libros básicos, pueden organizar todos los elementos y crear un proyecto de su propia autoría con explicaciones que se ajustan a su propio nivel de conocimiento de las matemáticas o de la ciencia de computación, etc. En general, el mito de la incompetencia de los estudiantes de primer o segundo semestre para abordar temas y problemas complejos por falta de "prerrequisitos» se puede revaluar fuertemente al ver a estos dos grupos de estudiantes producir análisis tan sofisticados como los que se encuentran en sus informes.

En el modelo ABP los estudiantes trabajan con usos reales de las matemáticas y no sólo con sus teorías abstractas. Ellos aprenden teoría matemática en un escenario más tradicional basado en un curso, pero muy a menudo sólo utilizan estas herramientas indirectamente en los informes de proyecto. Los cursos les presentan técnicas y herramientas que apoyan su aprendizaje en los proyectos. En este sentido, el formalismo matemático no se posiciona como la finalidad misma del aprendizaje, sino como un medio. Esto no significa, sin embargo, que los estudiantes de hecho no manejen los detalles del formalismo y que no sean diestros en ello. Lo que significa es que lo segundo sucede porque lo primero genera las razones fuertes para poder enfrentar las exigencias tradicionales del aprendizaje de las matemáticas universitarias.

Además, es importante tener en cuenta que los ámbitos de uso definidos por un problema que requiere la construcción de ensamblajes funcionales con las herramientas matemáticas y de otras disciplinas simplemente no podría encajar dentro de un curso de matemáticas que considere las necesidades de todos los grupos de estudiantes. En el modelo mixto de cursos clásicos de matemáticas y aprendizaje de las matemáticas a través de $\mathrm{ABP}$, se atiende tanto al entrenamiento 
sintáctico como a la competencia de usar y recontextualizar las matemáticas. Sin embargo, si los estudiantes de ingeniería no necesitan una comprensión profunda de alguna teoría matemática como tal -que podría argumentarse como muy importante en el caso de estudiantes de matemáticas- se podría decir que el modelo $\mathrm{ABP}$ es realmente suficiente para cumplir la mayoría de las metas de aprendizaje matemático para ingeniería. Lo que vemos aquí es que este tipo abierto de ambiente de ABP abre la posibilidad de que los estudiantes se involucren en un proceso de actividad de ensamblaje multidisciplinario de herramientas de conocimiento y artefactos propios de los campos que estudian. Así, las relaciones entre los conocimientos en la construcción de ensamblajes funcionales para responder a sus problemas potencian la competencia de actuar con los conocimientos. Los conocimientos del currículo -que formas tradicionales de currículo universitario presenta aisladamente- adquieren significado en su relación y función en los ensamblajes.

Los casos que hemos discutido por supuesto son apenas ejemplos elegidos entre muchos otros de los proyectos que cada semestre producen los estudiantes del primer ciclo de ciencias e ingeniería en la Universidad de Aalborg. El análisis de otros proyectos podría abrirnos el espacio para descubrir muchas más características del uso del modelo ABP. En los ejemplos presentados nos hemos enfocado sobre los resultados de aprendizaje relacionados con la brecha entre el formalismo y el uso de las herramientas matemáticas. A continuación nos gustaría considerar brevemente otra perspectiva de estos hallazgos sobre el modelo ABP de Aalborg que hemos solamente tocado de manera indirecta hasta ahora, a saber, el hecho importante de que este modelo promueve el aprendizaje activo como un principio conductor.

\section{El modelo ABP de Aalborg y los estudiantes como agentes}

¿Cómo podemos entender el enfoque de aprendizaje basado en problemas y organizado por proyectos con relación al aprendizaje activo en la educación universitaria? Es claro que el marco del ABP para el trabajo de los estudiantes toma en serio la manera como realmente se viven en la práctica la investigación, el desarrollo científico y la innovación. El significado de los datos, la teoría y el método en el modelo del aprendizaje basado en problemas entra en una mezcla compleja de procesos iterativos de conceptualizaciones que implican reformulaciones de problemas de investigación y nuevas conexiones entre diferentes campos de estudio, incluso conexiones transdisciplinarias. De esta manera, el modelo ABP ofrece un espacio de aprendizaje activo y participativo en el sentido de que deben hacerse conexiones y recontextualizaciones entre un número de teorías, posiblemente a partir de una variedad de disciplinas, y deben ajustarse en el abordaje de un problema concreto y en un informe de proyecto con una justificación propia, y con una clara conclusión para su audiencia.

Este aspecto del aprendizaje activo inherente al modelo $\mathrm{ABP}$ se relaciona con el trabajo que los estudiantes hacen frente a un contenido específico que debe ser aprendido. Sin embargo, hay otro aspecto importante del aprendizaje activo inherente al modelo ABP que debe mencionarse. Queremos enfatizar aquí los procesos de aprendizaje que tienen lugar en los grupos que trabajan en proyectos. En una situación de ABP, los facilitadores - profesores e investigadores de la 
Universidad que tienen a su cargo los cursos magistrales que reciben estos estudiantes- se reúnen con el grupo de estudiantes para conversar sobre qué hacer y cómo proceder. Su función es orientar y facilitar; su función no es dirigir ni decidir lo que los estudiantes deben hacer. Así que la mayor parte de los procesos de aprendizaje real tiene lugar cuando los estudiantes están solos en su grupo, sin el apoyo de los facilitadores. La mayoría de los grupos trabajan durante muchas horas en sus proyectos y algunos experimentan con distintas técnicas y procesos para optimizar su aprendizaje, ayudados también por sus facilitadores en este aspecto. En cada grupo deben considerarse muchos procesos de cooperación y aprendizaje que son propios de ambientes de trabajo reales. ¿Cómo deberíamos compartir la información que encontramos? ¿Quién debería escribir qué y cuándo? ¿Cómo podemos usar mejor un horario de trabajo? ¿Necesitamos alternar el liderazgo del grupo? A los estudiantes no se les dice categóricamente qué hacer en este aspecto, a pesar de que se les ofrece un curso donde se discuten distintas técnicas de colaboración, manejo de proyectos, y de reflexión sobre los procesos de elaboración de los proyectos. Todos los grupos tienen muy diferentes ámbitos y necesidades: algunos luchan con el trabajo ético de algunos miembros, otros luchan con el contenido que ha de incluirse en el informe -los científicos de la computación, por ejemplo, quieren programar algoritmos de los resultados matemáticos que desarrollan- y otros prefieren invertir su energía en experimentar con diferentes sistemas para compartir conocimiento, diferentes tipos de procesos de escritura, desarrollo de agendas para encuentros de grupo; incluso algunos desarrollan un wiki para su propio espacio de aprendizaje.

Todo esto significa que los estudiantes pasan por una experiencia de aprendizaje activo y participativo, no sólo en relación con el contenido disciplinar e interdisciplinar que requieren aprender, sino también en relación con el proceso mismo de trabajar en equipo en una tarea dada. El aprendizaje activo en este aspecto ofrece a los estudiantes un entorno de aprendizaje en el que se les invita a reflexionar y experimentar con su cooperación y comunicación de grupo de una manera que les ayuda a desarrollar competencias generales que son valiosas en muchos otros contextos de su vida profesional y personal.

\section{Conclusiones}

En lo que sigue destacamos, por una parte, algunas de las conclusiones que se pueden obtener con respecto al modelo ABP de Aalborg en la forma en que ha sido esbozado a través de los estudios de caso. Por otra parte, consideramos los procesos de aprendizaje activo. Algunas de las conclusiones son generales en su alcance, en tanto que otras tratan directamente con el aprendizaje de las matemáticas en la educación universitaria. Primero destaquemos algunas de las características generales del modelo ABP:

- El aprendizaje activo se logra permitiendo que los estudiantes trabajen sobre problemas que no están estrictamente determinados de antemano por sus profesores. Este tipo de autoría es el combustible principal que alimenta las reflexiones sobre cómo resolver un problema particular. Esto incluye involucrarse en la formulación de una problematización de un área que se quiere abordar, identificar un problema delimitado que se quiere trabajar, lo mismo que reflexionar sobre la metodología 
científica y el enfoque desarrollado para presentar el informe del proyecto. La experiencia muestra que estos aspectos de la generación de un producto científico son muy complejos y requieren de la ayuda de facilitadores que tienen experiencia en la investigación como parte de su trabajo cotidiano.

- En un entorno de aprendizaje que siga el modelo ABP, se posibilita que los estudiantes desarrollen muchas competencias de trabajo en equipo y de comunicación. Asegurar un espacio de aprendizaje en el que ciertamente surgen discusiones, reflexiones, diferencias de opinión, etc. implica el perfeccionamiento de la capacidad de los estudiantes para cooperar efectivamente, organizarse en equipos, reunirse y adquirir conocimientos.

Estos aspectos del modelo ABP son generales en su alcance pero, por supuesto, constituyen un ingrediente muy importante cuando se organiza el aprendizaje de las matemáticas. El sistema general ABP apoya la cooperación estrecha entre estudiantes, la interacción enfocada con facilitadores, y moldea los procesos de aprendizaje que tendrán lugar. Además de los rasgos generales, también hemos tocado algunos que tienen una influencia directa con respecto a la brecha entre el formalismo y los usos y recontextualizaciones de las matemáticas en otros campos de conocimiento.

- El contenido matemático en el que los estudiantes llegan a ser competentes es el que realmente tiene un valor de uso específico directo para un cierto programa educativo.

- Diferentes grupos de estudiantes pueden finalmente aprender diferentes porciones de las matemáticas dependiendo del proyecto que escriban, pero a su vez, se les ofrece la oportunidad de poder cerrar la brecha entre el formalismo y sus usos.

- A los estudiantes se les da la oportunidad de aprender que el uso de las matemáticas en otros campos de conocimiento no es un simple proceso de aplicación o de transferencia, sino que se trata de un proceso complejo de recontextualización dentro un escenario específico de práctica.

- El modelo ABP puede integrar matemáticas en un estudio interdisciplinario de un problema del mundo real.

- El modelo también abre las posibilidades para hacer meta-reflexiones y metateorizaciones que iluminan el papel de las matemáticas en la construcción de soluciones tecnológicas. Aquí la dimensión ética y social de las matemáticas se hace evidente. Este tipo de reflexiones hacen parte de las competencias de un profesional en el mundo actual (Skovsmose, 2008).

Estas conclusiones definen lo que podrían considerarse como algunos de los beneficios producidos al usar el modelo ABP para el aprendizaje de las matemáticas universitarias. Son variaciones de respuestas del modelo $\mathrm{ABP}$ al problema de cerrar la brecha entre el formalismo y los usos de las matemáticas: la relevancia de las matemáticas aprendidas, las matemáticas al servicio de resolver un problema dado, el modelaje matemático en un escenario de práctica dado y la posibilidad de usar matemáticas en estudios interdisciplinarios.

Habiendo establecido estas conclusiones, es desde luego importante abordar el hecho de que hay muchas discusiones educativas retadoras e interesantes que involucran el uso del modelo ABP como se ha esbozado. Durante los últimos años, el asunto de la evaluación individual de los 
estudiantes universitarios ha sido la prioridad de la agenda de las políticas educativas danesas. Debido al temor de que se colaran en el sistema de evaluación grupal algunos estudiantes que no habían trabajado comprometidamente en el proyecto -y que por lo tanto no habían alcanzado el nivel de competencia requerido en cada nivel- el gobierno decidió prohibir la realización de exámenes grupales como ha sido la tradición en la Universidad de Aalborg. ¿Cómo se puede decir cuáles estudiantes han contribuido realmente a la investigación y a escribir el informe del proyecto? La idea de evaluar a los estudiantes inscritos en el mismo semestre en la misma Universidad, pero sobre la base de informes de proyectos muy diferentes -ellos rara vez comparten el mismo contenido matemático aun si son estudiantes de matemáticas- sugiere que se requieren muchas consideraciones para garantizar una evaluación ajustada a los logros de cada estudiante.

Otro desafío interesante para el modelo ABP de Aalborg es la pregunta acerca del nivel de libertad que los estudiantes deberían tener para decidir sobre qué tema trabajar. Evidentemente los temas están limitados por el programa académico en cuestión, pero algunos programas de estudio tienen definiciones muy estrictas de lo que ha de aprenderse a partir de la escritura de un informe de proyecto dado en un cierto semestre de educación. De esta manera, los planeadores educativos tienen mucho que pensar con respecto al modelo ABP. En la práctica puede funcionar simplemente como un marco para escribir de una manera especial un contenido casi predeterminado o puede ser un marco para procesos extremadamente abiertos desde el principio, que dejan espacio para muchas de las ideas básicas de permitir que los estudiantes trabajen a través de un enfoque basado en problemas.

Otros retos interesantes incluyen problemas que conciernen a la diversidad en cuanto a los antecedentes culturales de los estudiantes, lo mismo que a las diferencias de edad e identidad entre miembros de grupos, etc., lo que abre el debate sobre los límites para la implementación el modelo $\mathrm{ABP}$ en diferentes modelos educativos y para diferentes tipos de programas. La Universidad de Aalborg aborda de maneras complejas todos estos asuntos y el modelo ABP se redefine constantemente, tanto a través de la práctica educativa como de la influencia de la investigación educativa sobre los muchos aspectos del uso del modelo ABP. Aquí hemos tratado de mostrar cómo el modelo es un marco benéfico para generar un tipo de aprendizaje de matemáticas que va más allá de las limitaciones del aprendizaje tradicional a nivel universitario. Nuestra perspectiva muestra cómo los estudiantes no sólo generan significado y participan activamente, sino que además muestran el desarrollo de la competencia para ensamblar conocimientos de varias disciplinas y generar nuevos artefactos funcionales apropiados para tales contextos problémicos. La posibilidad de operar dentro de esos diversos juegos de lenguaje es de hecho una de las mejores ganancias de un estudiante que será profesional en el mundo laboral tecnológico-científico de hoy.

\section{Referencias bibliográficas}

Abdulwahed, M., Jaworski, B., y Crawford, A. R. (2012). Innovative approaches to teaching mathematics in higher education: A review and critique. Nordic Studies in Mathematics Education, 17(2), 49-68. 
Barrows, H. S. (1986). A taxonomy of problem-based learning methods. Medical Education, 20(6), 481-486.

Beach, K. (1999). Consequential transitions: A sociocultural expedition beyond transfer in education. Review of Research in Education, 24, 101-139.

Bingolbali, E., y Monaghan, J. (2008). Cognition and institutional setting. En A. Watson y P. Winbourne (Eds.), New directions for situated cognition in mathematics education (Vol. 45, p. 233-259). Springer US.

Dahl, B., y Kolmos, A. (2015). Students' attitudes towards group based project exams in two engineering programmes. Journal of Problem Based Learning in Higher Education, 3(2), 62-79.

Flyvbjerg, B. (2001). Making social science matter: why social inquiry fails and how it can succeed again. Oxford, UK; New York: Cambridge University Press.

Hernández, C., Ravn, O., y Valero, P. (2015). The Aalborg University PO-PBL model from a sociocultural learning perspective. Journal of Problem Based Learning in Higher Education, 3(2), 16-36.

Knijnik, G. (2012). Differentially positioned language games: ethnomathematics from a philosophical perspective. Educational Studies in Mathematics, 80(1), 87-100.

Kolmos, A. (2008). Problem-based and project-based learning. En O. Skovsmose, P. Valero y O. R. Christensen (Eds.), University science and mathematics education in transition (p. 261280). New York: Springer.

Kolmos, A., Fink, F. K., y Krogh, L. (2004). The Aalborg PBL model. Aalborg: Aalborg University Press.

Krogh, L., y Jensen, A. A. (Eds.). (2013). Visions, challenges and strategies: PBL principles and methodologies in a Danish and global perspective. Aalborg: Aalborg University Press.

Latour, B. (1994). On technical mediation. Philosophy, sociology, genealogy. Common Knowledge, $3(2), 29-64$.

Lobato, J. (2006). Alternative perspectives on the transfer of learning: History, issues, and challenges for future research. Journal of the Learning Sciences, 15(4), 431-449.

Niss, M. (2002). University mathematics based on problem-oriented student projects: 25 years of experience with the Roskilde Model. En D. Holton, M. Artigue, U. Kirchgräber, J. Hillel, M. Niss y A. Schoenfeld (Eds.), The teaching and learning of mathematics at university level (Vol. 7, p. 153-165). Springer Netherlands.

Renshaw, E. (1991). Modelling biological populations in space and time. Cambridge; New York: Cambridge University Press.

Roth, W.-M. (2008). The gap between university and the workplace: Examples from graphing in science. En O. Skovsmose, P. Valero y O. R. Christensen (Eds.), University science and mathematics education in transition (p. 133-155). New York: Springer.

Schoenfeld, A. H. (1992). Learning to think mathematically: Problem solving, metacognition, and sense making in mathematics. En D. A. Grouws (Ed.), Handbook of research on Mathematics teaching and learning (p. 334-369). New York: Macmillan.

VV.AA. (2005). Fugleinfluenza [Gripe aviaria]. Aalborg: Basisår. Det teknisk-naturvidenskabelig Fakultet. Aalborg Universitet.

VV.AA. (2007). Rangordning af søgeresultater [Jerarquía de resultados de búsqueda]. Aalborg: Basisår. Det teknisk-naturvidenskabelig Fakultet. Aalborg Universitet. 
Vithal, R., Christiansen, I. M., y Skovsmose, O. (1995). Project work in university mathematics education. A Danish experience: Aalborg University. Educational Studies in Mathematics, 29(2), 199-223.

Vithal, R., y Valero, P. (2003). Researching mathematics education in situations of social and political conflict. En A. Bishop, M. A. Clements, C. Keitel, J. Kilpatrick y F. K. S. Leung (Eds.), Second international handbook of Mathematics education (Vol. 2, p. 545-592. Dordrecht: Kluwer.

Wittgenstein, L. (1997). Philosophical investigations. Oxford: Blackwell Publishers. 